\title{
THE IMPACT OF PHARMACEUTICAL CARE ON IMPROVING THE QUALITY OF LIFE IN PATIENTS WITH ALLERGIC RHINITIS
}

\section{Anna Todorova, ${ }^{1}$ Antoaneta Tsvetkova, ${ }^{2}$ Silvia Mihaylova, ${ }^{3}$ Kalina Andreevska, ${ }^{4}$ Antonia Kondova, ${ }^{5}$ Mariana Arnaoudova ${ }^{6}$}

\begin{abstract}
:
Introduction: Allergic rhinitis (AR) is a chronic disease with great social and economic impact that is largely undiagnosed and inadequately self-treated. Healthcare professionals such as pharmacists play a key role in recognizing and assessing the severity of AR, dispensing of OTC drugs, counseling of patients and in severe cases, referring them to health care specialists for further treatment.

Objective: This study explores the impact of pharmaceutical care and patient counseling on the self-management of seasonal AR.

Methods: The participating pharmacists follow the stepwise algorithm of ARIA (Allergic Rhinitis and its Impact on Asthma) Pharmacist's Guide. The effectiveness of the specialized pharmaceutical care is assessed by the changes in patients' quality of life (QOL) before and after the provision of patient counseling and pharmacist monitoring on patient self-management. The quality of life is measured with the help of the generic tool 12v2 SF.

Results: As a result of the provided pharmaceutical care, the analysis indicates improvement of all assessed aspects of patients' QOL. The analysis shows that the better scores of the indicators Physical functioning, Vitality, Physical health and Mental health are not accidental; rather, they are in a statistically significant correlation with the provided pharmaceutical care.

Conclusion: The study highlights the need for professional patient counseling and the education of patients to perform adequate disease management and improve their quality of life.
\end{abstract}

UDC Classification: 615.1; DOI: http://dx.doi.org/10.12955/cbup.v5.1064

Keywords: allergy, rhinitis, pharmacists;

\section{Introduction}

Pharmacists act as intermediaries between physicians and patients in ensuring effective prevention and treatment of chronic diseases. Pharmaceutical care encompasses patient counseling, disease prevention, monitoring, and evaluation of the treatment (Dessing, 2000). We should emphasize that the pharmacist's role is to assist patients in self-treatment with OTC drugs only when disease control is achievable. The collaboration between patients, pharmacists, and physicians aims at establishing the optimal therapy and managing good disease control.

\section{Literature review}

AR is a chronic disease with great social and economic impact due to numerous factors: its high prevalence, the disease-related impairment of patients' quality of life, patients' inability to perform various professional and academic activities, the economic burden of AR, and its comorbidity asthma and other allergic diseases (Baiardini et al., 2006, Bousquet et al., 2008). The contemporary holistic approach to socially significant chronic diseases encompasses not only the medical treatment, but also the management of disease symptoms, prevention of disease complications and improvement of the quality of life of patients (Meltzer et al., 2012).

AR is largely undiagnosed and inadequately treated, thus being a predisposing factor for the subsequent development of asthma (Pawankar et al., 2011).

To prevent disease progression, the World Health Organisation (WHO) launched various initiatives for health education and provides recommendations for the treatment of AR through ARIA guideline. (Allergic Rhinitis and Its Impact on Asthma). In compliance with ARIA guidelines pharmacists, being the most accessible health care professionals, have to identify allergic rhinitis, assess the symptom

\footnotetext{
${ }^{1}$ Faculty of Pharmacy, Medical University of Varna, annitodorova@abv.bg

${ }^{2}$ Education and Research Center, Medical College of Varna, Medical University of Varna, antoaneta_cvetkova@abv.bg

${ }^{3}$ Education and Research Center, Medical College of Varna, Medical University of Varna, s_mihaylova@mail.bg

${ }^{4}$ Medical University of Plovdiv, Faculty of Pharmacy, andreevska@abv.bg

${ }^{5}$ Faculty of Pharmacy, Medical University of Varna, antoniakondova@mail.bg

${ }^{6}$ Medical University-Varna, marnaudova@ hotmail.com
} 
severity, and provide patient counseling while dispensing OTC drugs when disease control is maintainable. (ARIA Pocket Guide for Pharmacists, 2003).

According to the disease severity and symptom frequency, ARIA guidelines classify AR as intermittent or persistent. Depending on the severity of the disease and the extent to which it affects the quality of life of the patient, AR is classified as mild or moderate/severe (Brozek et al., 2010). ARIA classification reflects patient's condition and is the determinant factor for the selection of the therapeutic regimen and the assessment of the maintained disease control (Pawankar et al., 2011). The stepwise approach that pharmacists need to adopt in the selection of alternative pharmacological treatments and patient counseling according to the AR type is part of the algorithm developed by ARIA (Fig.1).

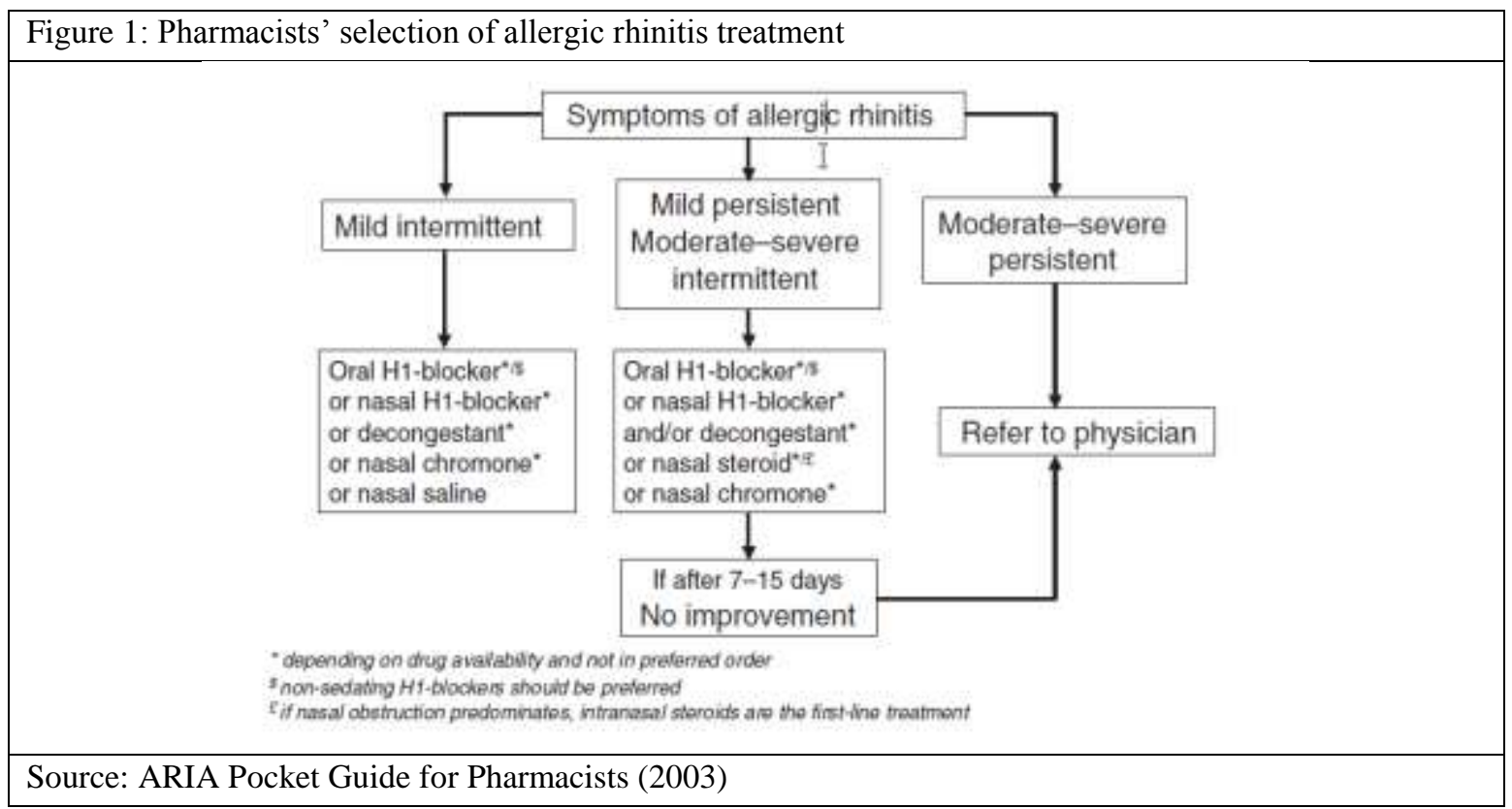

According to ARIA and WHO, disease control in patients with AR can be maintained through patient education, health promotion and disease prevention (avoidance of specific allergens or triggering factors), a healthier environment, and drug therapy and immunotherapy (Allergic Rhinitis and Its Impact on Asthma). The role of the pharmacist is directed towards improving public health through the promotion of a healthy life style (Guidelines on Good Pharmacy Practice, 2009). As health care professionals, pharmacists do not simply handle and dispense medications, but they also provide valuable information about the disease symptoms that can be subject to self-medication, potential adverse drug reactions, contraindications, and special treatment of patients with chronic diseases. (Tsvetkova et al., 2015).

The objective of the study is to analyze the results of pharmaceutical care provided to patients with allergic rhinitis who practice self-medication by monitoring as well as evaluate the quality of life before and after the intervention of the pharmacist.

Data and methodology: Pharmaceutical care was provided in compliance with ARIA guidelines for pharmacists, including individual training, patient counseling at the dispensing of appropriate OTC products, monitoring the effectiveness of the treatment, and in cases of no positive treatment outcome, referring the patients to a physician.

The effectiveness of the pharmaceutical care was analyzed by assessing the quality of life in patients before and after the provided consultation and the treatment monitored by the pharmacist.

The study was conducted in community pharmacies in the city of Varna. Pharmaceutical care was provided to patients with AR who sought professional advice at the corresponding pharmacy, selfmedicated with OTC drugs, and followed no therapy regimen prescribed by a physician at the time of the study.

The study spanned over the pollen season (from March to June) when the acute phase of the disease was more likely to be triggered. 
The survey participants were adults 20 to 45 years of age. This specific age group has the highest morbidity rate, and therefore it was in the focus of our study.

The patients' quality of life was assessed with the help of the generic tool SF12 v2 (the shorter version of SF 36). SF-12v2 Health Survey is a questionnaire validated for Bulgaria, intended to assess the physical health, the mental health, and the social functions within a 4-week recall period. SF-12v2 is a health survey, consisting of 12 questions that address eight domains in order to evaluate the physical and mental health of patients and their social functions. It provides two composite scores which assess the physical health and the mental health. The SF-12V2 questionnaire is based on the data obtained from the Quality Metric 2009 Norming Study conducted in the USA in 2009 by The National Research Corporation (NRC). It served as a basis for the scale used in this survey. The norm for the studied health composite summaries was set at 50 points with a minimum value of 20 pts. and a maximum value of 80 pts. The higher the score, the better the quality of life.

After a prior consent and instructions on how to fill out the questionnaire, participants replied in writing to self-assess their quality of life.

The study used a Student's t-test for assessing the statistical significance of the results obtained after the provided pharmaceutical care. Hypothesis testing was performed with a significance level set at 0.05 .

Results and Discussion: The baseline survey involved 71 patients with pronounced AR symptoms seeking medical advice in the pharmacy. A follow-up survey of patients QL was performed after the provided pharmaceutical care, including patient counseling and patient education, dispensing of the appropriate OTC product, and health status monitoring in accordance with ARIA algorithm. The follow-up SF12v2 test involved 63 patients. Eight of the baseline participants dropped out from the follow-up test because they did not meet the survey inclusion criteria and were referred to a medical specialist by the corresponding pharmacist.

The summarized results of the assessment of the Physical Component Summary (PCS) and the Mental Component Summary (MCS), before and after the provision of pharmaceutical care, compared to the norm are presented in Figure 2 and Figure 3.

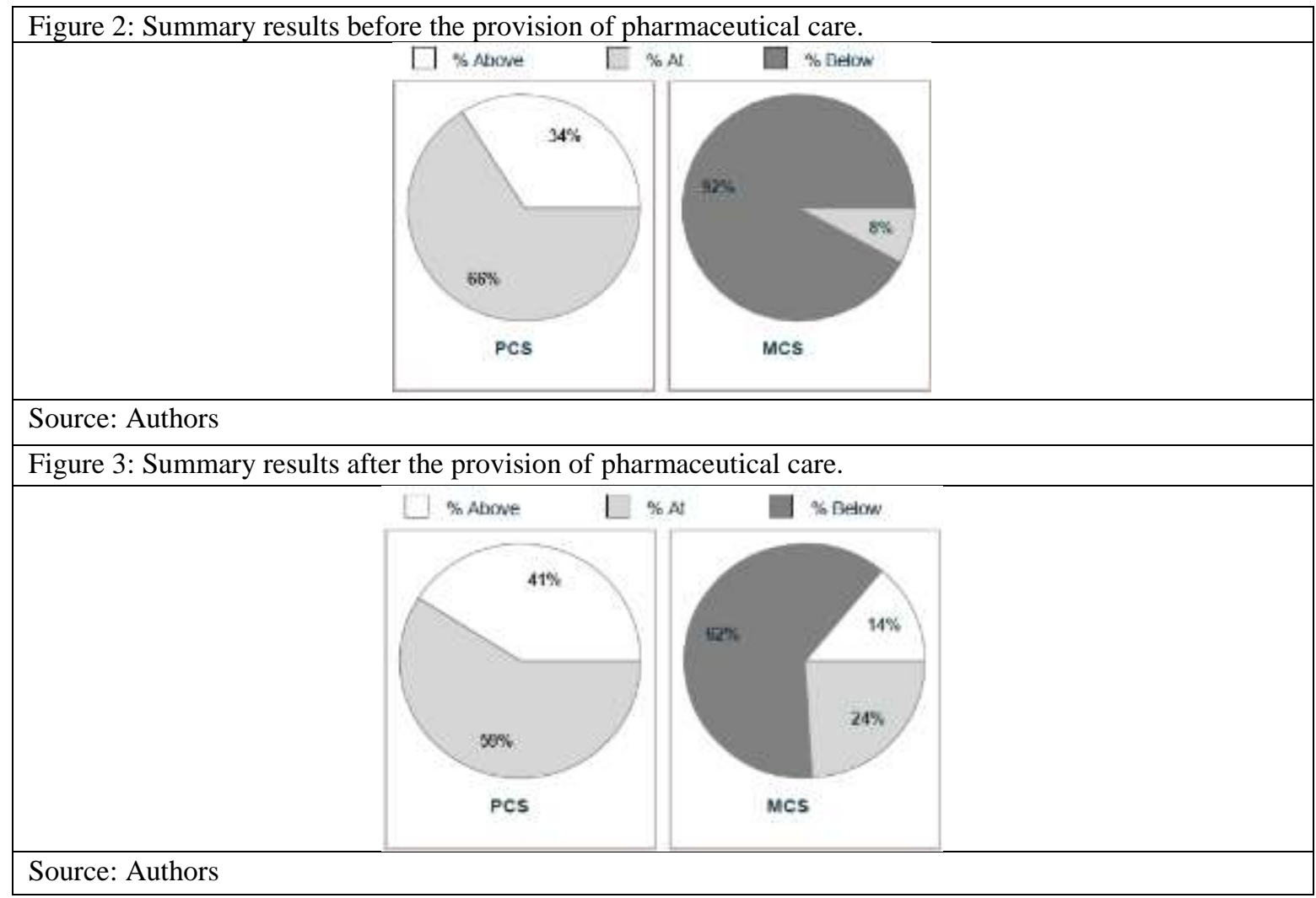

In $66 \%$ of the respondents in the baseline test, the Physical Component Summary is within the norm and for the remaining $34 \%$ it is above the norm. Highly prevalent are the patients (92\%) whose mental 
wellbeing is below the norm in the survey scale. The mental health is within the norm in only $8 \%$ of the respondents.

Following the provided pharmaceutical care, the Physical Component Summary (PCS) scores better; it is within the norm in 59\% of the respondents and above the norm in the remaining $41 \%$. Impressive is the improvement of the Mental Component Summary (MCS) - it is below the norm in only $63 \%$ of the patients, which is a $40 \%$ decrease in comparison with the baseline test. The share of patients $(24 \%)$ with MCS within the norm is increasing. In the follow-up test, the MCS score is above the norm in $14 \%$ of the patients, whereas there were no patients registered in this category during the baseline test. Figure 4 displays the share of patients that are at risk of depression before and after the provision of pharmaceutical care correspondingly.

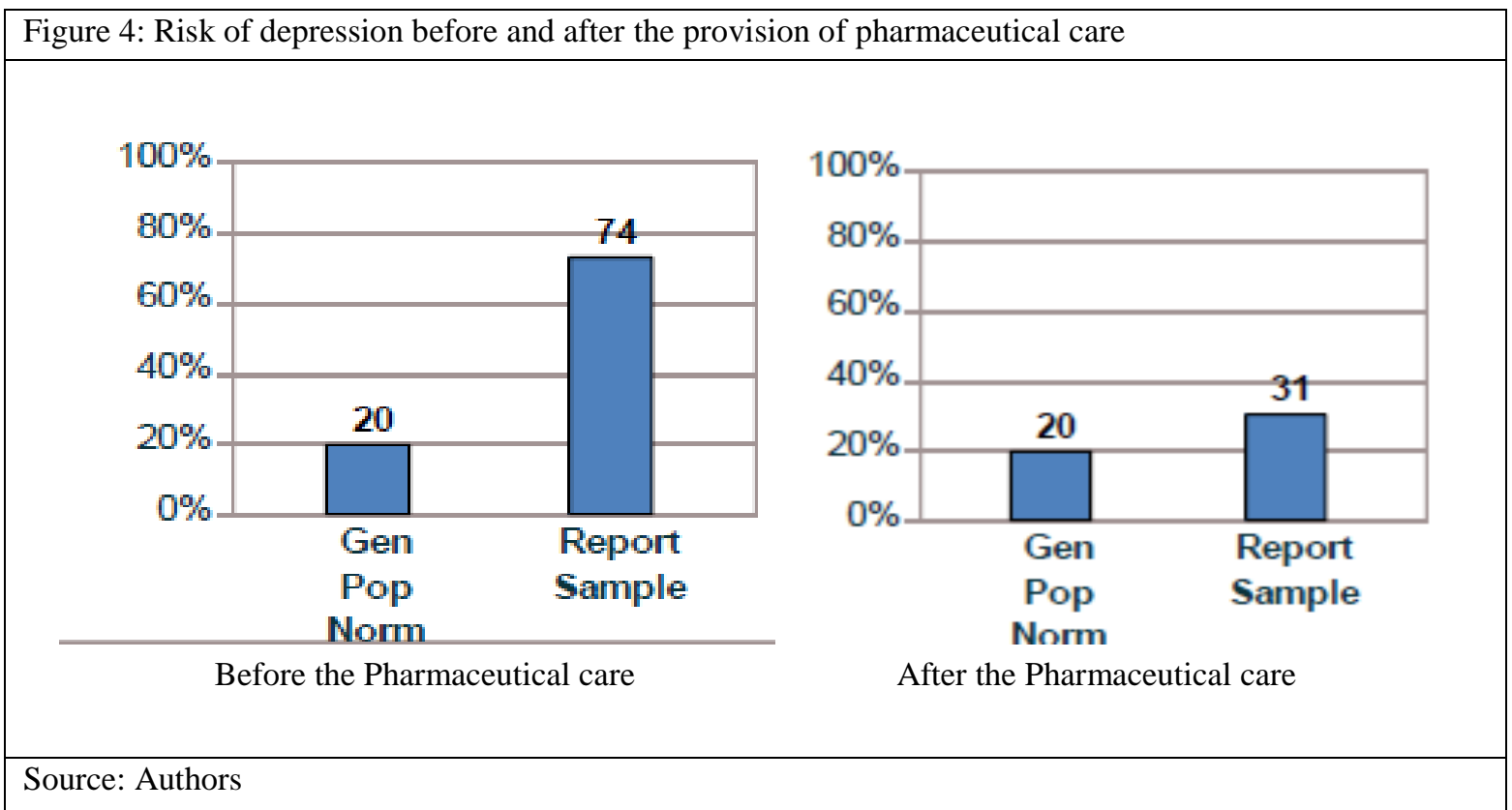

The general population norm for high-risk patients was set at $20 \%$ by the Quality Metric 2009 Norming Study, the primary goal of which was the development of representative norms for survey tools such as the SF-12V2 questionnaire.

The baseline test results show that $74 \%$ of the respondents are at risk of depression. As a result of the provided pharmaceutical care, there is a significant decrease of $43 \%$ in the share of patients that are at risk of depression; the total share falling to $31 \%$. In the baseline test, the depression risk indicator was $54 \%$ above the general population norm, whereas in the follow-up test, it declines to $11 \%$ above the norm. The comparison between the baseline and the follow-up survey shows that the provided pharmaceutical care resulted in a remarkable decline of 50\% in the share of patients who are at risk of depression. Other studies have found that symptoms such as mood swings, anxiety, and depression are much more frequent in patients with allergic rhinitis than in healthy controls (Cuel et al., 1999). A previous research on the QOL in patients with seasonal AR who resort to self-medication has found that symptoms of AR and the disease-related complaints are risk factors for the emotional well-being, social functioning, and mental health of AR patients (Todorova et al., 2015).

Figure 5 shows the comparative analysis of the results from the tests conducted before and after the provision of pharmaceutical care.

All indicators for physical, psychological, and social health score higher in the QL follow-up survey. Most pronounced is the change in the Vitality indicator with an increase of 11 points. The increase in the rest of the indicators is within the range of 3-5 points. The results are similar for the BP, GH, and SF indicators (a 3-points increase) and for the PF (a 5-points increase). The higher score of the Vitality indicator in the follow-up test evidences the QL improvement associated with the provided pharmaceutical care. We assume that patient-centred care and effective communication between pharmacists and patients enhance the adequate self-treatment and improve patients' mental and physical wellbeing 


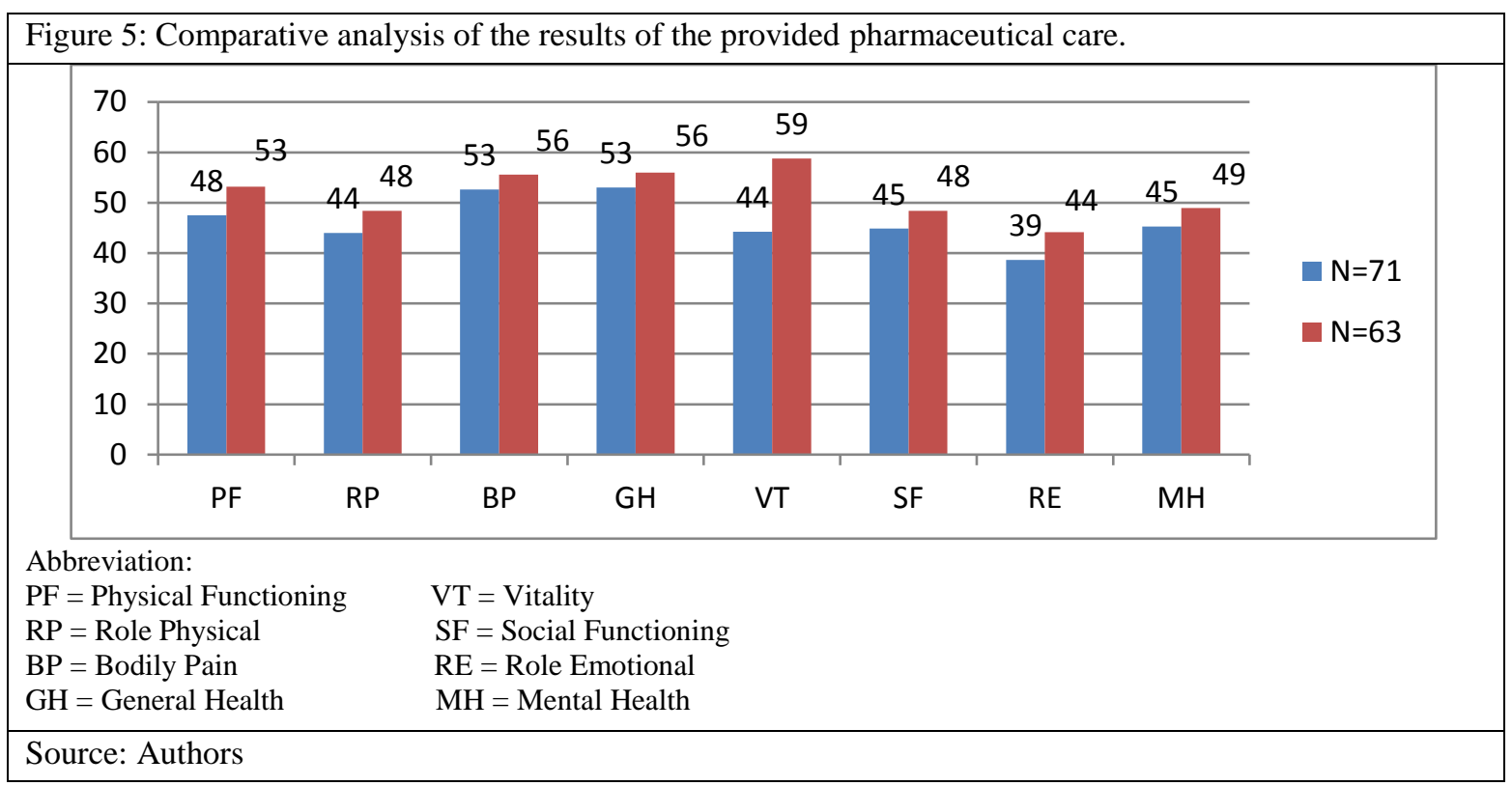

The statistical significance of the improvement data in the QOL indicators as a result of the provided pharmaceutical care is analyzed and the results are summarised in Table 1.

Table 1: Statistical significance of the improvement data as a result of the provided pharmaceutical care

\begin{tabular}{|l|l|l|l|l|l|l|l|l|l|l|}
\hline & PF & RP & BP & GH & VT & SF & RE & MH & PCS & MCS \\
\hline $\begin{array}{l}\mathrm{N}=7 \\
1\end{array}$ & 47.53 & 43.99 & 52.65 & 53.04 & 44.22 & 44.87 & 38.64 & 45.30 & 51.53 & 41.05 \\
\hline $\begin{array}{l}\mathrm{N}=6 \\
3\end{array}$ & 53.19 & 48.40 & 55.58 & 56.02 & 58.75 & 48.43 & 44.15 & 48.91 & 55.69 & 47.40 \\
\hline $\mathrm{n}$ & 71.00 & 71.00 & 71.00 & 71.00 & 71.00 & 71.00 & 71.00 & 71.00 & 71.00 & 71.00 \\
\hline $\mathrm{n} 1$ & 63.00 & 63.00 & 63.00 & 63.00 & 63.00 & 63.00 & 63.00 & 63.00 & 63.00 & 63.00 \\
\hline $\mathrm{s}$ & 7.46 & 9.40 & 4.47 & 7.21 & 10.68 & 7.33 & 12.81 & 4.45 & 6.36 & 2.87 \\
\hline $\mathrm{s} 1$ & 5.41 & 7.79 & 4.16 & 7.07 & 9.52 & 7.83 & 10.23 & 7.16 & 4.47 & 7.22 \\
\hline $\mathrm{t}$ & 1.97 & 1.16 & 1.55 & 0.95 & 3.27 & 1.07 & 1.08 & 1.40 & 1.71 & 2.70 \\
\hline tkp & 1.64 & 1.64 & 1.64 & 1.64 & 1.64 & 1.64 & 1.64 & 1.64 & 1.64 & 1.64 \\
\hline & $\begin{array}{l}\text { Significa } \\
\text { nt }\end{array}$ & $\begin{array}{l}\text { insignific } \\
\text { ant }\end{array}$ & $\begin{array}{l}\text { insignific } \\
\text { ant }\end{array}$ & $\begin{array}{l}\text { insignific } \\
\text { ant }\end{array}$ & $\begin{array}{l}\text { significa } \\
\text { nt }\end{array}$ & $\begin{array}{l}\text { insignific } \\
\text { ant }\end{array}$ & $\begin{array}{l}\text { insignific } \\
\text { ant }\end{array}$ & $\begin{array}{l}\text { insignific } \\
\text { ant }\end{array}$ & $\begin{array}{l}\text { significa } \\
\text { nt }\end{array}$ & $\begin{array}{l}\text { significa } \\
\text { nt }\end{array}$ \\
\hline
\end{tabular}

Source: Authors

According to the null hypothesis, the changes are due to random processes alone. The alternative hypothesis states that there is a statistically significant difference, and the changes are consequent upon the pharmaceutical care. The Hypothesis testing was performed with a significance level set at 0.05 .

The analysis shows that the improvement in the composite scores for physical health and mental health (PCS and MCS), as well as in the Physical functioning indicator and in the Vitality indicator is not a random process. There is a statistically significant dependence of this improvement on the pharmaceutical care provided according to ARIA algorithm. The statistically significant improvement in the PCS and MCS in patients with AR highlights the need for patient training and specialized pharmaceutical care.

Low public awareness of allergies as serious chronic diseases has been identified as a major health issue. Patients suffering from respiratory allergies are not fully aware of the importance of the allergies and the complications associated with the disease progression. Patients, especially those with milder forms of allergies, tend to adapt to their condition or resort to self-medication without consulting a physician (Bousquet, Cauwenberge, Khaltaev, 2003). According to data published, just $45 \%$ of patients with allergic rhinitis in Europe seek medical advice or treatment (Canonica et al., 2007). 
Patient education and environmental control measures are effective mechanisms for AR management. Self-monitoring and deliberate avoidance of triggering environmental factors are the first steps towards the control of respiratory allergies. Studies examining the impact of patient education and specialised pharmaceutical care indicate better QL and fewer disease complications in patients who have been subject to pharmaceutical care and have participated in specially designed educational programs.

The optimal therapeutic regimen is a multifaceted process. Its efficacy depends on the adequate selection of drugs, drug dosage and treatment duration, which is accomplishable only with the participation of a health professional. We should be aware that a common behaviour pattern of patients with diagnosed AR is to resort to self-treatment with no prior medical consultations or allergy tests.

Notwithstanding the improvement in patients' QL, their attention should be drawn to the fact that pharmacotherapy is effective for treating only the symptoms of the disease. In no way can pharmacological treatment affect the cause and the disease triggering factors. The contemporary approach to disease treatment requires an unambiguous proof of the allergic nature of patients' complaints and allergen detection. The effective management of socially significant chronic diseases should address not simply the disease symptoms, but the cause and the trigging factors as well.

\section{Conclusion}

Timely treatment of AR should be considered a crucial component of asthma management and an essential part of the disease control and prevention. The study confirms the belief that AR constitutes a serious threat to patients' wellbeing. The effect of the pharmaceutical care provided in compliance with ARIA guidelines indicates that pharmacists can successfully enhance the adequate selfmanagement and improve the QL in patients with intermittent and light persistent AR.

Pharmacists are a valuable resource that can be managed more effectively to make their unique contribution in protecting and enhancing public health and wellbeing even better.

\section{References}

Allergic Rhinitis and Its Impact on Asthma (ARIA) http://www.whiar.com

Allergies in America. A telephone survey conducted in 2500 adults with allergic rhinitis. Health star communications. Inc., in partnership with Shulman, Ronca and Bucuvalas, Inc. Allergies in America: A landmark survey on nasal allergy sufferers. Executive summary. 2006.

ARIA Pocket Guide for Pharmacists. In Edition 2003.

Baiardini I, Braido F, Brandi S, Canonica GW. Allergic diseases and their impact on quality of life. Ann Allergy Asthma Immunol. 2006; 97:419-428;

Bousquet J, Khaltaev N, Cruz A, et al. Allergic rhinitis and its impact on asthma update. Allergy 2008; 63Supl 86: 9 - 160.

Bousquet J, van Cauwenberge P, Khaltaev N. Allergic rhinitis and its impact on asthma (ARIA)- Executive summary. Allergy 2003;57:841-855

Brozek JL, Bousquet J, Baena-Cagnani CE et al. Allergic Rhinitis and its Impact on Asthma (ARIA) guidelines: 2010 revision. J Allergy Clin Immunol 2010; 126: 466-476.

Camelo-Nunes IC, Sole D. Allergic rhinitis: indicators of quality of life. J Bras Pneumol 2010; 36(1); 124 - 33.

Canonica GW, Bousquet J, Mullol J et al. A survey of the burden of allergic rhinitis in Europe. Allergy 2007; 62 Suppl 85: $17-25$

Cuel B, Wamboldt M, Borish L. et al. Economic consequences of comorbid depression, anxiety and allergic rhinitis. Psychosomatics, 1999; 40: 491 - 496.

Dessing RP. Ethics applied to pharmacy practice. Pharm World Sci 2000; 22: 10-16

Guidelines on Good Pharmacy Practice, Approved by the Minister of Health ref.No 62-00-70-08/11.03.2009

Meltzer EO, Gross GN, Katial R, Storms WW. Allergic rhinitis substantially impacts patient quality of life: findings from the Nasal Allergy Survey Assessing Limitations. J Fam Pract. 2012;61(2):5-10.

Pawankar R, Canonica G, Holgate S, Lockey R. WAO White Book on Allergy 2011-2012. In Edition Milwakee, Wisconsin, USA: WAO 2011.

Todorova A., Tsvetkova A., Arnaoudova M., Dimitrov M., Researching the quality of life in patients with seasonal allergic rhinitis practicing self- treatment, WJPPS, 2015, 4 (12):1-11

Tsvetkova A., Todorova A., Tsvetkov L.' Mihailova S., Dimitrov M., The role of the pharmacist in achieving therapeutic efficacy and cost effectiveness in the management of allergic rhinitis" International Journal of Information Research and Review 2015; 2(4). 593-596, 